\title{
Nouveau procédé pour la stabilisation de la marche de centrales hydro-électriques avec chambre d'équilibre
}

\author{
Etude théorique et résultats expérimentaux
}

\author{
New method for stabilizing \\ the operation of hydroelectric power \\ stations having surge tanks
}

\author{
PAR \\ M. CUENOT) \\ ET \\ INGÉNIEUR E.P.F. ZURICH \\ A. GARDEL \\ INGÉNIEUR E.P.U.L., LIC, ÈS SC.
}

\begin{abstract}
Nonvelle méthode de stabilisation des oscillations du plan d'eau de chambres d'óquilibre pror asservissement de la charge électrique a la charge hydraulique. Résultat d'essais effectues $\dot{a}$ la centrale d'oelberg (canton de Fribourg, Suisse). Ces essais ont confirmé les résultats auxquels avaient conduit les études théoriques : il a été possible de maintenir une marche stable de l'usine malgré une réduction de la section de la chambre d'équilibre de 300 à $7 \mathrm{~m}^{2}$. Le choia des dimensions $\dot{a}$ donner $\dot{a}$ une chambre d'équilibre peut donc être libéré des servitudes que lani impose la condition de stabilité des oscillations dues au réglage.
\end{abstract}

\begin{abstract}
New method of stabilizing water surface oscillations in surge tanks by establishing a connection between the electrical loal and hydratlic head. Result of tests made at the Oelberg power station (Canton of Friburg, Switzerland). These tests confirme the results obtained in the theoretical studies; it is possible to maintain stabilized operation of the power station although the section of the surge tank is reduced from 300 to $7 \mathrm{sq}$. meters. The dimensions of a surge tank can therefore be chosen without paying attention to the stability of oscillations caused by gonerning action.
\end{abstract}

\section{INTRODUCTION}

En 1910 déjà, D. Thoma a exposé quelle était la répercussion de la condition de stabilité des oscillations du plan d'eau d'une chambre d'équilibre sur le choix des dimensions à donner à cette chambre.

Cette « condition de Тнома » a été amendée par la suite pour tenir compte de certains effets plus ou moins secondaires. Ces différents calculs avaient en commun comme base l'hypothise que la puissance fournie par Ia centrale considérée est constante. Par suite de l'action du réglage de vitesse de la turbine, une élévation du plan d'eau dans la chambre d'équilibre provoque en compensation une fermeture de la turbine, ce qui accentue cette élévation et conduit à une marche instable, si la section de la chambre d'équilibre est insuffisante.

La cause de cette instabilité est supprimée si, à la suite d'une élévation du niveau de l'eau dans la chambre d'équilibre, la puissance débitée par le groupe est augmentée de façon à supprimer, sinon à réduire la fermeture de la tur- 
bine, et vice versa en cas de baisse du niveau d'eau. Cette variation de la charge électrique en fonction de la charge hydraulique peut être réalisée par un asservissement de la tension du générateur au niveau d'eau dans la chambre d'équilibre si le réseau a un caractère ohmique, ou par un asservissement de la fréquence à co nivenu d'eau si le réseau alimente des moteurs (1 $\dot{a} 3)\left({ }^{\star}\right)$.

En première approximation, on peut admettre que, par rapport à la duréc des oscillations du plan d'eau de la chambre d'équilibre, le réglage de vitesse agit instantanément; cependant, pour certaines installations spécialement défavorables à cet égard, lorsque la période des oscillations du réglage de vitesse est du même ordre de grandeur que celle des oscillations de la chambre d'équilibre, cette hypothèse n'est pas valable. Il faut alors prendre en considération la stabijité de l'ensemble de l'installation, ensemble constitué par le groupe, sa chambre d'équilibre, sa conduite forcée, son réglage de vitesse et le réseau qu'il alimente.

Il est connu que la stabilité du réglage de vitesse peut être améliorée lorsque la charge est ohmique (cas le plus défavorable), par un asservissement tension à la fréquence, asservissement qui fait en sorte que la charge électrique augmente lorsque la fréquence augmente et vice versa quand la fréquence diminue (4).

La figure 1 représente le schéma de principe de ces deux modes d'asservissement. En séric avec le potentiomitre $P_{1}$ qui permet d'ajuster à main la valeur de consigne du réglage de la tension, sont branchés la résistance de réglage

(*) Jes chiffres indiqués entre parentheses se référent à la bibliographic donnée en annexe.

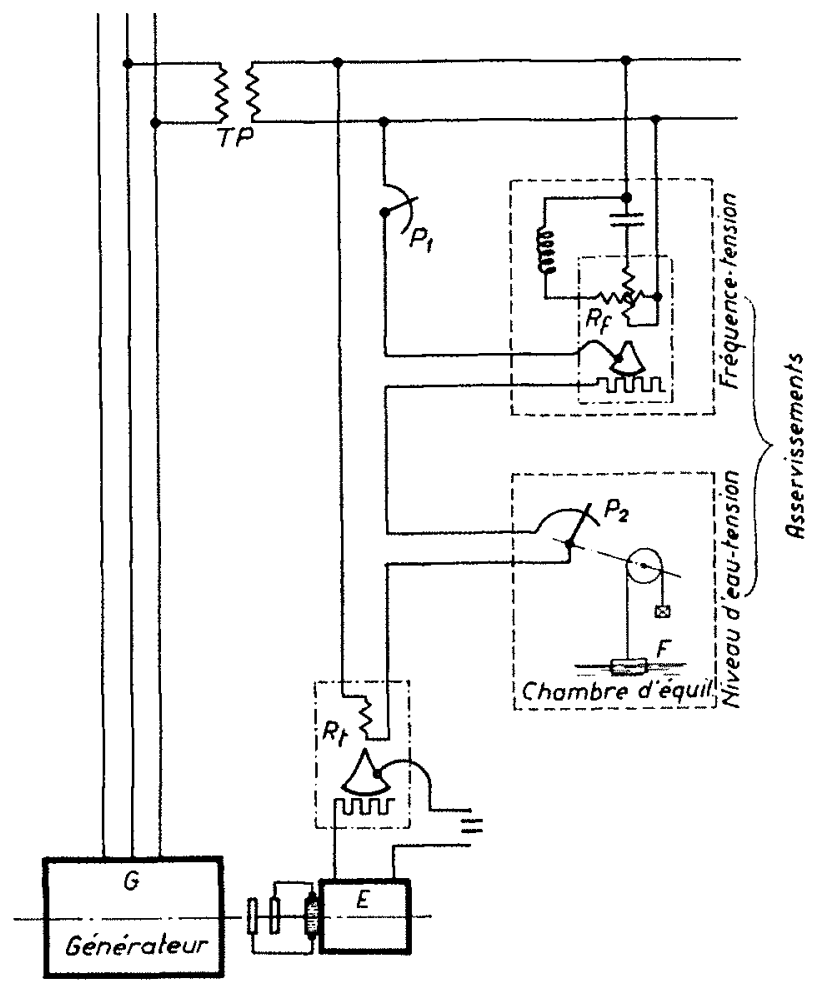

Firi, 1. - Schéma de principe des asservissements.

du régulateur de fréquence $R_{\text {f }}$ et le potentiomitre $\mathrm{P}_{2}$ dont le contact mobile est entrainé par le flotteur $F$ qui mesure le niveau d'eau dans la chambre d'équilibre.

Si l'on veut rendre la tension indépendante des variations permanentes de la fréquence ou du plan d'eau dans la chambre d'équilibre, ces deux asservissements doivent être rendus passagers par des dispositifs qui ne sont pas représentés sur la figure 1.

\section{I. - DETERMINATION THEORIQUE DES CONDITIONS DE STABILITE}

La figure 2 représente le principe de la disposition d'un groupe hydro-électrique arec cham- bre d'équilibre. Lal lurbine T, alimenté par l'intermediaire de la conduite forcé $c$ e et de la

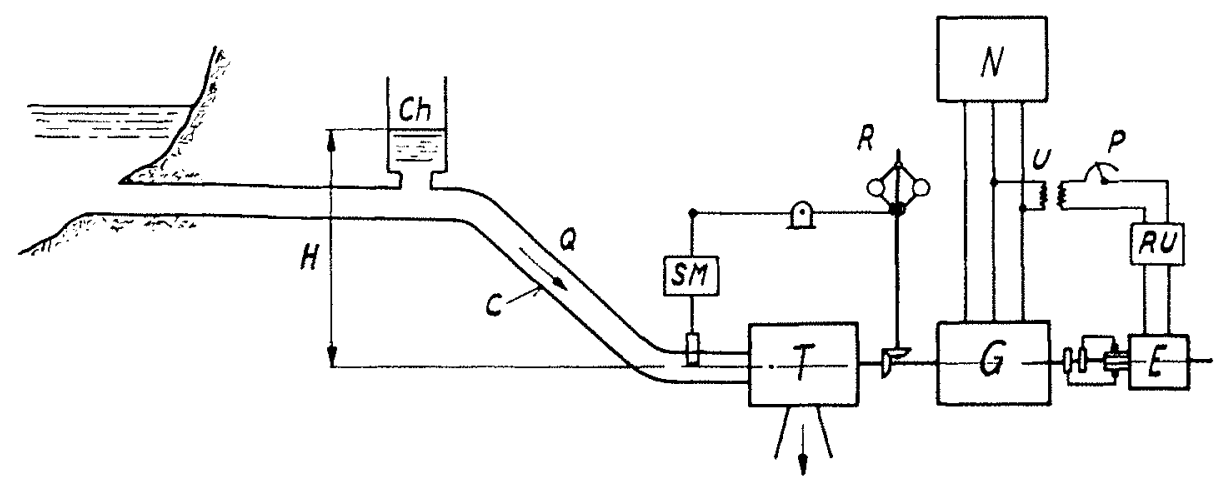

Fıa. 2. - Principe de la disposition d'un groupe hydro-électrique arec chambre d'équilibre. 
chambre d'équilibre $\mathrm{Ch}$, entraine le généraleur $G$ qui débite son énergie sur la charge N. La vitessc angulaire $\omega$ est mesurée par le régulateur $R$ qui commande, par l'internédiaire du servo-moleur SM, l'ouverture de la turbine. La tension U est mesurée par le régulateur de tension RU qui ajuste l'excitation du générateur, par l'intermédiaire de l'excitatrice $\mathrm{E}$. Ce dispositif est caractérisé par les variables suivantes:

- Le débit de l'eau dans la conduite forcée $Q$;

- L'ouverture de la turbine Y;

- La hauteur du plan d'eau dans la chambre d'équilibre $\mathrm{H}$;

- La vitesse angulaire du groupe $\omega$;

- Le couple moteur $\mathrm{M}_{t}$;

- Le couple résístant $\mathrm{M}_{e}$;

- La charge $\mathbf{N}$.

Nous considérons de faibles variations par rapport à un état de régime que nous choisissons comme état de référence et que nous caractérisons par l'indice zéro. Nous admettons d'une part que le réglage de vitesse est astalique avec une stabilisation au moyen d'un accélérometre, et d'autre part que seul le coup de hélier en masse doit être pris en considération.

Le comportement dynamicue de l'installation est caractérisé par le système d'écjuations différentielles suivant (2):

$$
\begin{aligned}
& \mathrm{T}_{g} \Delta x+2 p_{0} \mathrm{~T}_{g} \Delta \dot{x}+\Delta x=-\mathrm{T}_{g} \Delta \dot{q}-2 p_{0} \Delta q \\
& \Delta q=\Delta y+1 / 2\left(\Delta h+\Delta h_{1}\right) \text { avec } \Delta h=\frac{\Delta x}{h_{0}} \\
& \Delta h_{1}=-2 \mathrm{~T}_{r} \Delta \dot{q} \\
& \Delta(1)+m \Delta \dot{\theta}=-\mathrm{T}_{r} \Delta \dot{y} \\
& \mathrm{~T}_{q} \Delta \dot{\omega}+\Delta m_{t}=\Delta m_{t} \\
& \Delta m_{t}=\Delta y+3 / 2\left(\Delta h+\Delta h_{1}\right)+\Delta \omega \mathrm{T}_{g} \beta \\
& \Delta m_{\varepsilon}=\Delta n+\mathrm{K}_{b} \Delta h+\Delta a \mathrm{~T}_{g} z
\end{aligned}
$$

avec :

$\Delta x=\left(\mathrm{H}-\mathrm{H}_{0}\right) / Z_{*}=$ ecart relatif du plan d'eau de la chambre d'équilibre.

$Z_{*}=$ irandeur de comparaison $=Q_{0} \sqrt{\mathrm{L} / g f \mathbf{F}}$.

$\mathrm{Q}_{0}=$ débit de régime.

$\mathrm{L}=$ longueur de la galerie d'amenée.

$f=$ section de la galerie d'amenée.

$\mathbf{F}=$ section de la chambre d'equilibre.

$T_{g}=\sqrt{ }$ CF/gT temps caractéristique de la galerie d'amenée. $g=$ constante d'accélération de la pesanteur. $p_{0}=\left(\mathrm{P}_{0}+\mathrm{E}_{0}\right) / \mathrm{Z}_{*}=$ perte de charge relative.

$\mathrm{E}_{0}=$ hauleur représentative de l'énergie cinétique à l'insertion de la chambre, pour le débit nominal.

$\Delta q=\left(\mathrm{Q}-\mathrm{Q}_{0}\right) / \mathrm{Q}_{0}=$ écart relatif du débit dans la conduite forcée.

$\Delta y=\left(Y-Y_{0}\right) / Y_{0}=$ écart relatif de l'ouverture de la turbine.

$\Delta h=\left(\mathrm{H}-\mathrm{H}_{0}\right) / \mathrm{H}_{0}=$ écart relatif de la charge hydraulique dû $\dot{a}$ une variation du plan d'eau dans la chambre d'équilibre.

$\Delta h_{1}=$ écart relatif de la charge dî au coup de bélier.

$h_{0}=\mathrm{H}_{0} / Z_{*}=$ chute relative.

$\mathrm{T}_{c}=\mathrm{L}_{c} \mathrm{~V}_{0} / 2 g \mathrm{H}_{0}=$ temps caractérislique de la conduite forcée.

$\mathrm{L}_{c}=$ longueur de la conduite forcée.

$\mathrm{V}_{0}=$ vitesse d'écoulement de l'eau dans la conduite forcée à l'état do régime.

$\mathrm{T}_{r}=$ temps caractéristique du dispositif de riglage.

$m=$ dosage accélérométrique.

$\mathrm{T}_{a}=\mathrm{PD}^{2} \mathrm{\Omega}_{0}{ }^{2} / 366 \mathrm{~N}_{0}=$ constante d'accélération du groupe.

$\Omega_{0}=$ vitesse de rotation nominale.

$\Delta n=\left(\mathrm{N}-\mathrm{N}_{0}\right) / \mathrm{N}_{0}=$ variation relative de la charge.

$\Delta m_{1}=\left(M \quad-M_{n}\right) / M_{n}=$ écart relalif du couple risislant.

$\Delta m_{t}=\left(\mathrm{M}_{t}-\mathrm{M}_{1}\right) / \mathrm{M}_{4}=$ ecart relatif du couple moteur.

Is $x_{t}=$ pente aux environs du point de régime du couple résistant.

$\operatorname{tg} \alpha_{t}=$ pente aux environs du point de régime du couple moteur.

$\mathrm{K}_{h}=\Delta_{n} / \Delta h=$ coefficient d'asservissement de la charge électrique au niveau d'eau.

Nous déterminons l'équation caractéristique du système, détermination qui est grandement facilitée par l'usage du calcul opérationnel et qui conduit à l'expression suivante avec l'općrateur (s) comme variable :

$$
1+\frac{1+s m}{s \mathrm{~T}_{r}} \frac{2}{a+s \mathrm{~T}_{o}}
$$

$\left\lceil\frac{1+s \mathrm{~T}_{c}+\left(1-\mathrm{K}_{h}\right) \varphi_{q h}}{2\left(1+s \mathrm{~T}_{c}\right)-\varphi_{q h}}-\frac{3}{2}-\frac{s \mathrm{~T}_{c}}{1+s \mathrm{~T}_{c}}\right\rceil=0$ 
avec :

$$
\varphi_{q h}=\frac{1}{h_{0}} \frac{\stackrel{2}{-} p_{0}+s \mathrm{~T}_{g}}{1+2 s \mathrm{~T}_{\eta} p_{0}+\mathrm{s}^{2} \mathrm{~T}_{y^{2}}}
$$

et $a=\operatorname{tg} \alpha_{e}-\operatorname{tg} \alpha_{t}=$ coefficient de sensibilite des couples aux écarts de fréquence.

$\operatorname{tg} \alpha_{c}$, et par conséquent $a$, peuvent être ajustés à volonté au moyen d'un asservissement fréquence-tension doni nous admettons laction instantanée. Il est possible, aux dépens d'une complication des calculs, de tenir compte de la constante de ce dispositif d'asservissement.

Jans le cas général, les racines de l'équalion caractéristique sont complexes; pour que le réglage soit stable, il faut et il sulfit que la partie réelle de ces racines soit négative. Celle condition se vérifie au moyen d'un des critìres de stabilité connus : Honwriz, NyQuis'r ou I.roNHARD. Dans notre cas, l'équation caractéristique étant du $5^{\circ}$ degré et son expression analytique étant connue, c'est le critère de LEONHARD qui apparaît comme le plus commode.

Les figures $3 a$ et $3 b$ donnent un exemple des courbes de LeONHARD ainsi obtenues et les valeurs numériques pour lesquelles elles ont été calculées; ces valeurs numériques correspondenl au cas de la centrale d'Oelbers (Fribourg), où des essais ont été effectués, essais dont les résultats sont relatés dans le deuxiome chapitre de cet exposé.

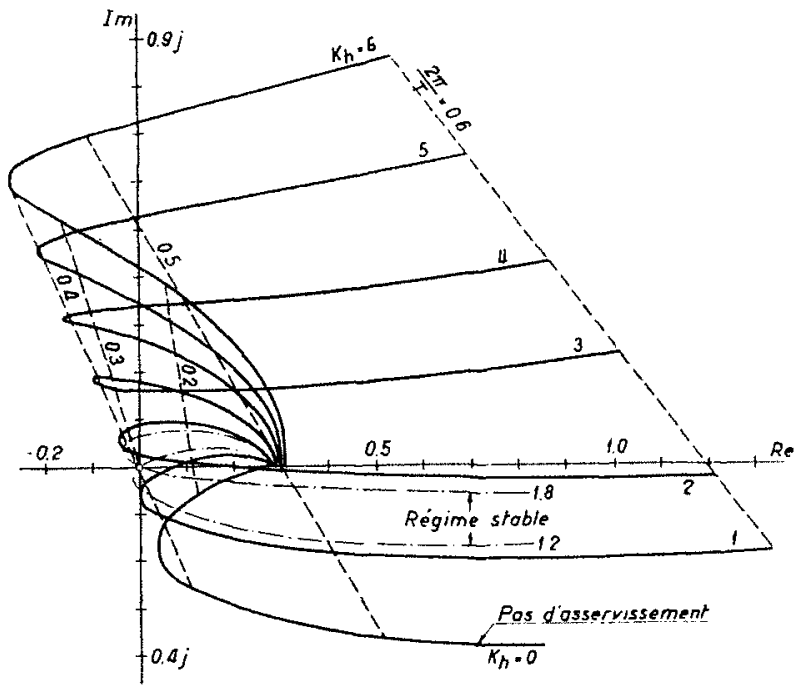

Fı. $3 a .-$ Facteur $a=0$.
Les conditions de stabilite ainsi determines peuvent être représentèes dans un diagramme en fonction des coefficients d'asservissement $a$ et $\boldsymbol{K}_{h}$ caractérisant les deux genres d'asservissement. La figure 4 donne un exemple de ce diagramme avec le temps caractéristique de réglage ' $T_{r}$ comme paramitre. On oblient des courbes en $\checkmark$ renversies qui découpent le diagramme en deux zones. A droite de ces courbes, le réglage est stable, à rauche il est instable.

La figure 5 donne la forme de la zone de stabilité pour différents types de centrales:

La figure 5 a se rapporte au cas admis géneralement oil les deux genres de stabiliti fohambre d'équilibre el réglage vitesse) peuvent ètro considérés indépendamment l'un de l'autre.

Dans ce cas particulier, les conditions de stabilité sont les suivantes:

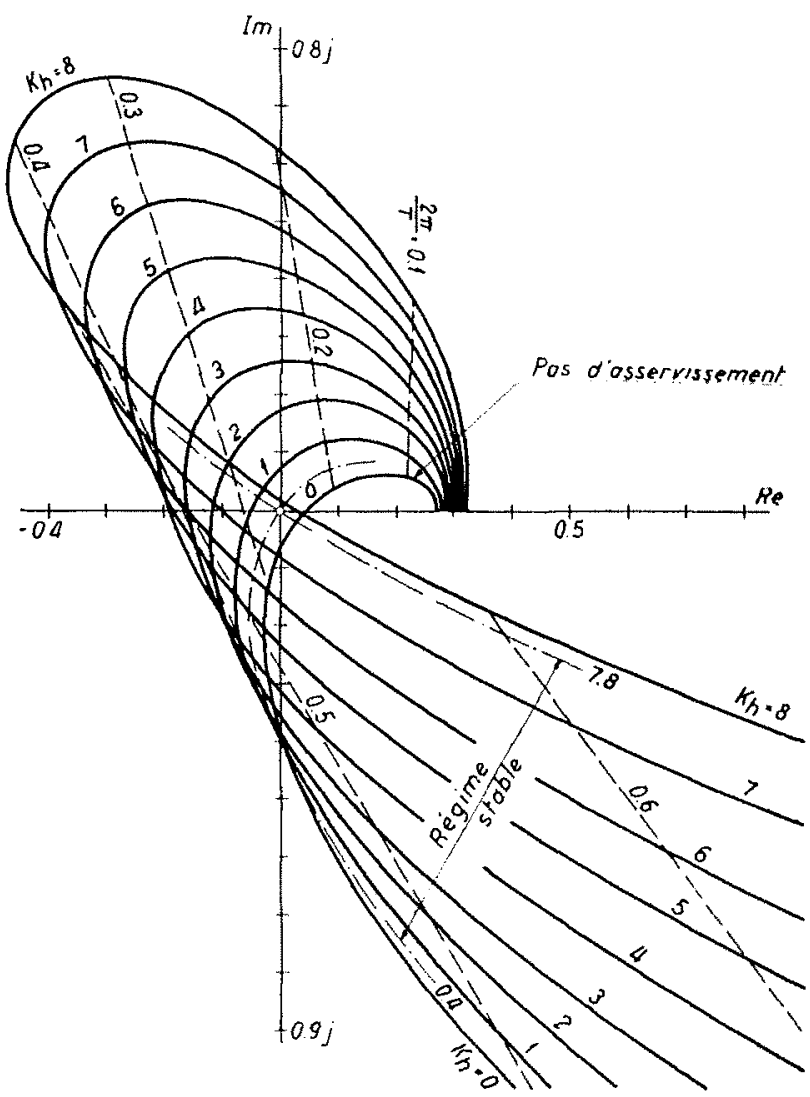

Fia. $3 b .-$ Facteur $a=2$.

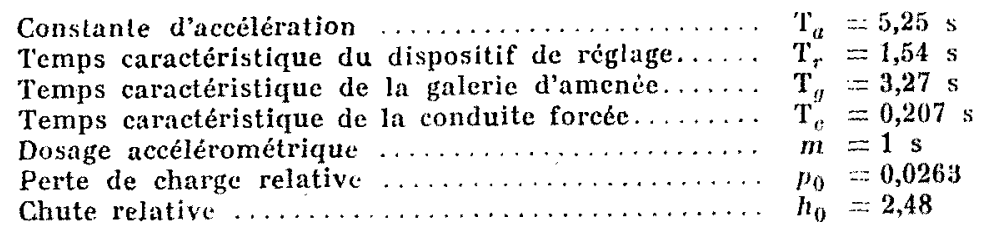

Fig. 3. - Détermination des conditions de stabilité it ladide de courbes de leonhard. 


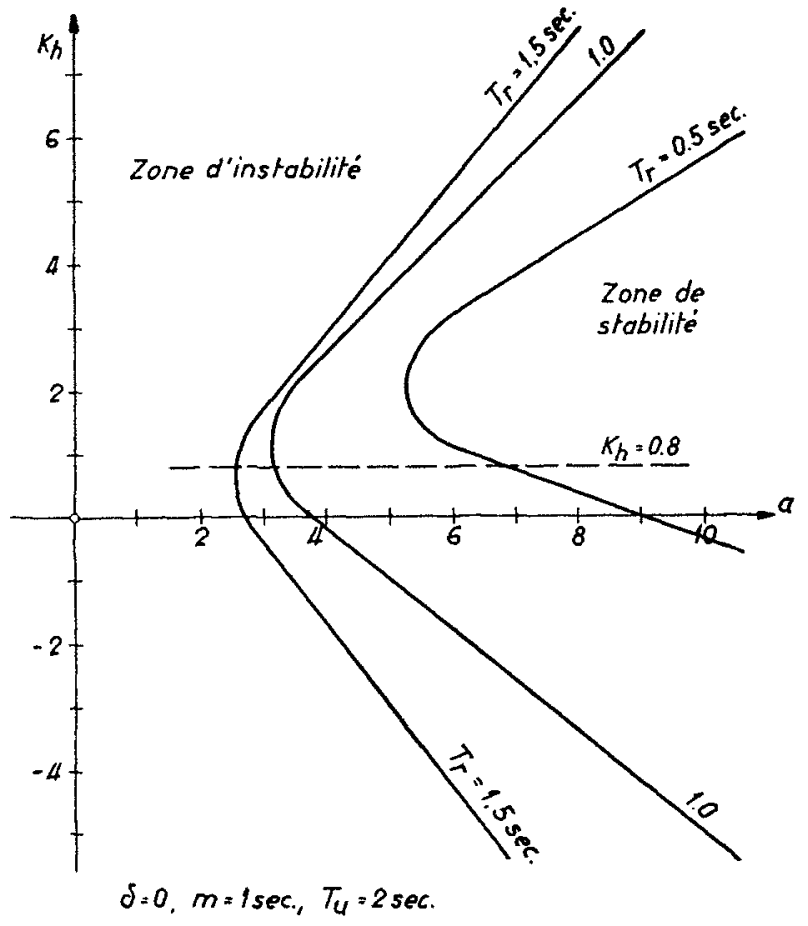

Fili. 4 - Conditions de stabilité en fonction des coefficients d'asservissement.

Parametre $=$ constante de temps $T_{r}$ du dispositif de réglage

- pour le réglage de vitesse (avec niveau d'eau constant):

$\left(a \mathrm{~T}_{c} \mathrm{~T}_{r}+\mathrm{T}_{a} \mathrm{~T}_{r}-2 m \mathrm{~T}_{c}\right)\left(a \mathrm{~T}_{r}-2 \mathrm{~T}_{c}+m\right)$

$$
-\mathrm{T}_{e} \mathrm{~T}_{a} \mathrm{~T}_{r}>0
$$

- pour la chambre d'éfuilibre (avec réglage de vilesse instantané):

$$
2 p_{01} h_{0}+\left(\mathbf{K}_{h}-1\right)>0 .
$$

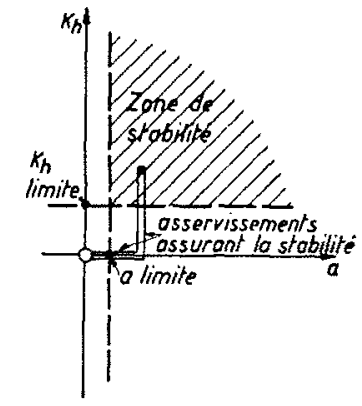

$5 a$

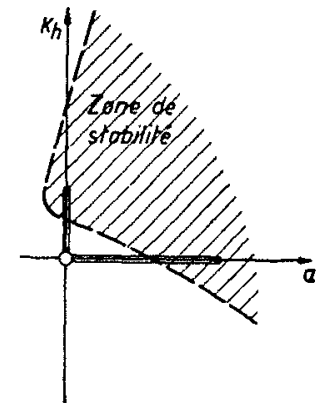

$5 b$
Lorsque I'on supprime l'asservissement au niveau d'eau $\left(K_{h}=0\right)$, on retrouve la condition bien connue de Thома :

$$
2 p_{0} h_{0}-1>0
$$

La figure $5 b$ s'applique dans le cas où ces deux genres de stabilité doivent être pris en considération simultanément selon la méthode de calcul que nous avons exposée.

La figure $5 c$ concerne le cas parliculier ou les périodes des deux genres d'oscillation sont très proches l'une de l'autre. Nous voyons que dans ce dernier cas il existe une valeur optimum du coefficient d'asservissement $K_{k}$. Si cet asservissement est trop fort ou trop faible, le réglage devient instable.

Il résulte de ces considérations théoriques que même pour une section de la chambre d'équilibre très inférieure à la section donnce par la condition de Tнома, on peut obtenir une marche stable à condition d'asservir judicieuscment la charge électrique au niveau d'eau de la chambre d'équilibre et à la vitesse.

MM. Gaden et Borel ont montré qu'un même effet de stabilisation pouvait être obtenu par un asservissement de la charge électrique à l'ouverture de la turbine (5). Cette méthode a l'avantage de supprimer liı nécessité d'une télémesure du plan d'eau, mais présente l'inconvénient de nécessiter un «filtre » pour faire la différence entre les variations de l'ouverture dues à une variation du niveau dans la chambre d'équilibre et celles qui sont dues à une variation de la consommation.

Fic. 5. - Condition de stabilite pour différents genres de centrales hydro-ejectriques avec chambre d'équilibre.

\section{II. - CONTROLE EXPERIMENTAL DU NOUVEAU MODE DE STABILISATION}

Des essais ont été entrepris sous la direction du Laboratoire d'Hydraulique de l'EPUL pour contrôler expérimentalement l'efficacité de la méthode de stabilisation qui fait l'objet de cet exposé.
Ces essais ont été effectués à la centrale d'Oelberg (canton de Fribourg, Suisse).

Le choix de cette centrale a été dicté par le fait que l'un de ses groupes était muni d'une chambre d'équilibre différentielle. En obturant 


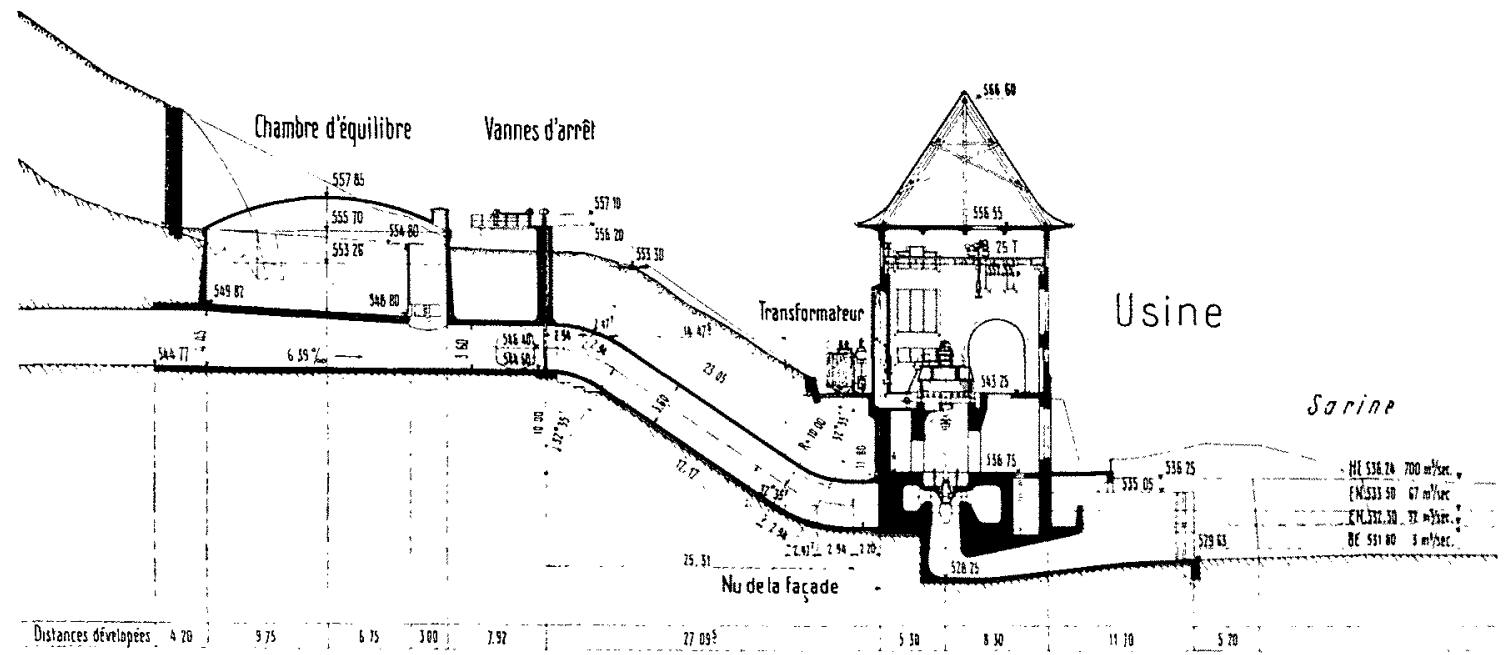

Fia. 6. - Profil en long de la centrale d'Oelberg.

par des vantaux les orifices (visibles sur le profil en long de l'installation donné par la figure 6) faisant communiquer le puits à la chambre proprement dite, on réduisait ainsi la section de la chambre d'équilibre $\left(300 \mathrm{~m}^{2}\right)$ à la seule section du puits $\left(7 \mathrm{~m}^{2}\right)$, section bien inférieure à la section de Tнома $\left(30 \mathrm{~m}^{2}\right)$. Les caractéristiques du groupe utilisé pour les essais sont les suivantes :

Puissance nominale............. $7500 \mathrm{ch}$

Débit nominal................. $32 \mathrm{~m} \%$

Chute maximum ............... $20,8 \mathrm{~m}$

Longueur de la galerie d'amenéc..... $270 \mathrm{~m}$

Section de la galerie d'amenée...... $18,3 \mathrm{~m}^{2}$

Longueur de la conduite forcée...... $47 \mathrm{~m}$

Section de la conduite forcée....... 10,2 $\mathrm{m}^{2}$

Turbine du type Kaplan, vitesse...... $250 \mathrm{t} / \mathrm{mn}$ Moment de giration du groupe $\mathrm{PD} 2$. .. $170 \mathrm{tm}^{2}$
Des essais préliminaires ont eu lieu en dicenbre 1951. Les résultats de ces essais ont été négatifs, en ce sens que malgri un asservissement de la charge électrique au niveau d'eau, il n'a pas été possible d'oblenir une marche stable. Ces essais préliminaires ont permis de constater que la période des oscillations dans la chambre d'équilibre élait très proche de celle du rí glage de vitesse (de 18 à $20 \mathrm{~s}$ ). Ice probleme de la stabilité du dispositif devail done etre traité dans son ensemble (chambre d'équilibe + rislinge de vitesse).

Les essais principaux ont eu lien en juin 1952. Les asservissements de la charge au niveau d'eau dans la chambre d'équilibre et à la fréquence ont té ajustés selon les valeurs auxquelles avait conduit l'étude théorique.
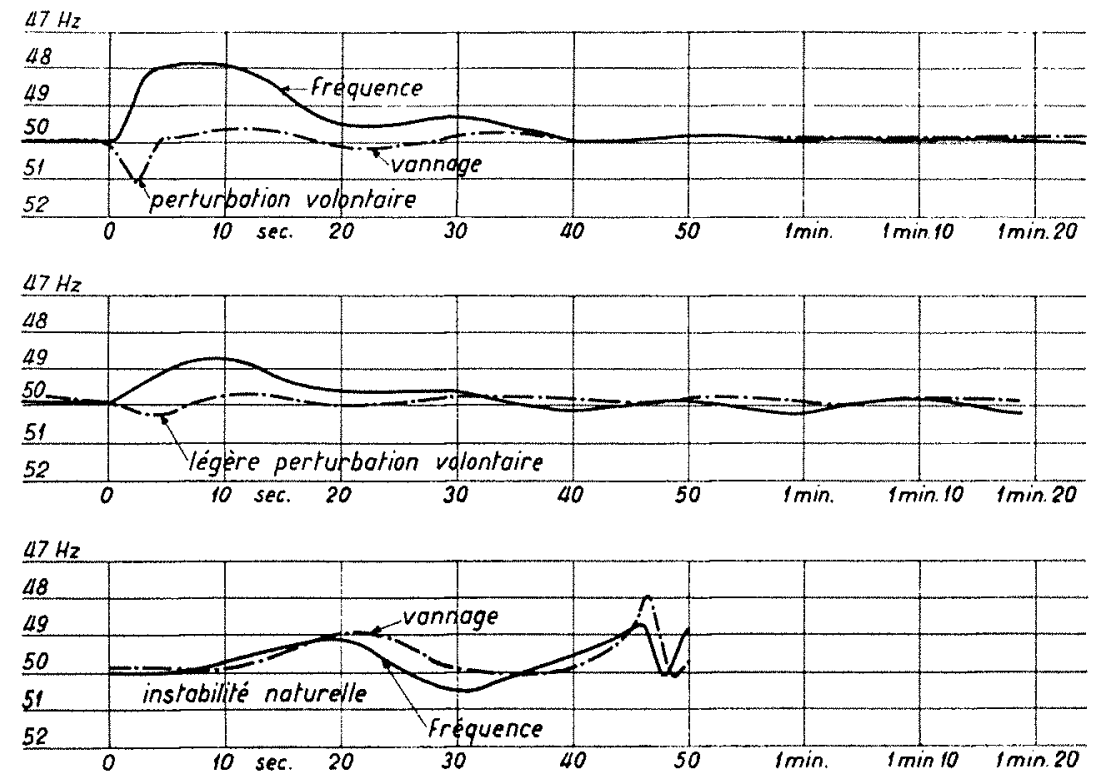

Essai 10

Fic. 7. - Enregistrements de la fréquenec et du vannage lors des essais d'Oelberg (juin 1952). 
Lors de ces essais, le vannage de la turbine itait soumis à une perturbation volontaire puis le systime était abandonné à lui-même.

La variation du vannage et cello de la vitesse du groupe ont été enregistrées; la figure 7 donne un exemple des enregistrements qui ont ité effectués pour lrois cas particulièrement caractéristiques.

La figure 8 récapilule le résultat des essais. Les ronds noirs correspondent à un réglage stahle, les ronds blanes à un réglage instable. Les ronds noirs cerclés correspondent au cas limile d'oscillations entrelenues. La surface hachurecorrespond à la zone limite telle que l'indiquent les calculs et compte tenu de l'imprécision avec laquelle sont connus certains des facteurs numériques.

On voit que, malgré la section utile de la chambre d'équilibre réduite dans la proportion de 40 à 1 , une marche parfaitement stable de l'installation a été obtenue, ceci malgré des conditions particulièrement défavorables dues à la période relativement très courte des oscillations de la chambre d'équilibre. Le principe de la stabilisation par asservissement de la charge électrique à la fréquence et au niveau d'eau a été confirmé expérimentalement. La concordance quantifative avec les résultats obtenus par voie théorique apparaît comme satisfaisante si l'on tient compte des hypothèses simplificatrices nécessitées par le calcul analytique et de l'imprécision avec laquelle plusieurs des facteurs numériques sont connus.

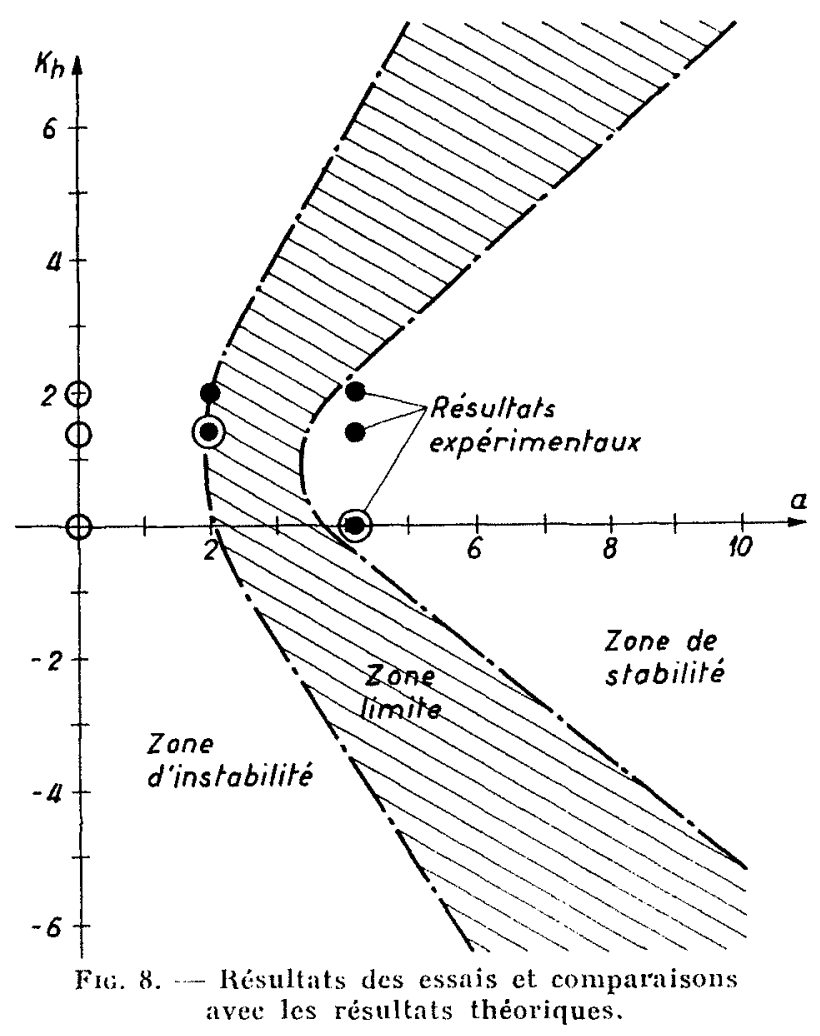

\section{CONCLUSION}

Les considerations théoriques confirmées par les calculs ont montré que, grâce à un asservissement judicieux de la charge électrique au niveau d'eau de la chambre d'équilibre et à la fréquence, une marche stable d'une centrale hydro-électrique avec chambre d'équilibre peut être obtenue, quelle que soit la dimension de cette chambre d'équilibre. Il n'est donc plus nécessaire dans ces conditions de prendre en considération la condition de stabilité pour fixer les dimensions d'une chambre d'équilibre.

Cette stabilisation est, il est vrai, obtenue aux dépens d'une tenue moins rigoureuse de la tension ou de la fréquence.

Nous avons cependant établi que les écarts de tension et de fréquence qui résultent de ce mode de stabilisation sont pratiquement négligeables (1). Ils ne se produisent que dans le cas exceptionnel de la marche isolée.

On sait que si une centrale avec chambre d'équilibre marche en parallele avec d'autres cen- trales et que la puissance de cette centrale est inférieure au tiers de la puissance totale du réseau, la stabilité de marche est obtenue quelle que soit la section de la chambre d'équilibre (6). Par suite du développement de l'interconnexion entre réseaux électriques, cette condition est pratiquement toujours réalisée. Cependant, toute centrale peut être amenée une fois ou l'autre à débiter son énergie sur un réseau isolé. Il n'apparaît pas économique d'assurer sa stabilité dans ce cas exceptionnel en augmentant spécialement dans ce but les dimensions de la chambre d'équilibre si le même résultat peut être obtenu par les dispositifs de réglage relativement très bon marché qui ont été décrits dans cet exposé.

Cette méthode est un exemple d'une solution par voie électrique apportée à un problème de génie civil et de la collaboration toujours plus étroite qui doit s'établir lors de l'élaboration de nouvelles centrales entre les ingénieurs civils, mécaniciens et électriciens. 


\section{Bibliographie}

1. M. Cuénod et A. Gander. -.. - Stabilisation des oscillations du plan d'eau dans la chambre d'équilibre $\gg$. Bul. techn. de la S.R., 1950, "1" 16.

2. M. Cuḱon el A. Ganben. -- " Stabilité de la marche d'une centrale hydro-électrique avec chambre d'équilibre, compte tenu des calactéristiques dynamiques du réglage de vitesse ». Bul. lechn. de la S.R., $1952, \mathrm{n}^{\circ} 24$.

3. M. Cuéxod et A. Ganner. - \& Essai de stabilisation du réglage d'un groupe bydro-électrigue muni de chambre d'équilibre ». Bul. techn. de la S.R., 1953, $n^{\circ} 16$ et 17 .
4. H. Kentaka. -... « La stabilisation du réglage de la vitesse des jroupes générateurs à charge indépendante de la fréquence $\gg$. Revae Rromn-Boveri, juin-juillet 1947 .

5. D. Gabex et L. Bonlit. ... \& Influence de la loi de variation de la puissance sue la condition de stabilité de Thoma $\$$. Bul. techn. de la S.R., 1951, $\mathrm{n}^{\circ} 9$.

6. J. Caldane et D. Gadex. - « De la stabilite des installations munies de chambre d'équilibre $\%$. Schw. Bauzpilung, 30-7-1927.

Adresse des auteurs:

M. Cúxon, C/o Ofinco, 1, rue de la Cité, Genève, Suisse.

A. Gardel, 13, av. Wamery, Lausanne, Suisse.

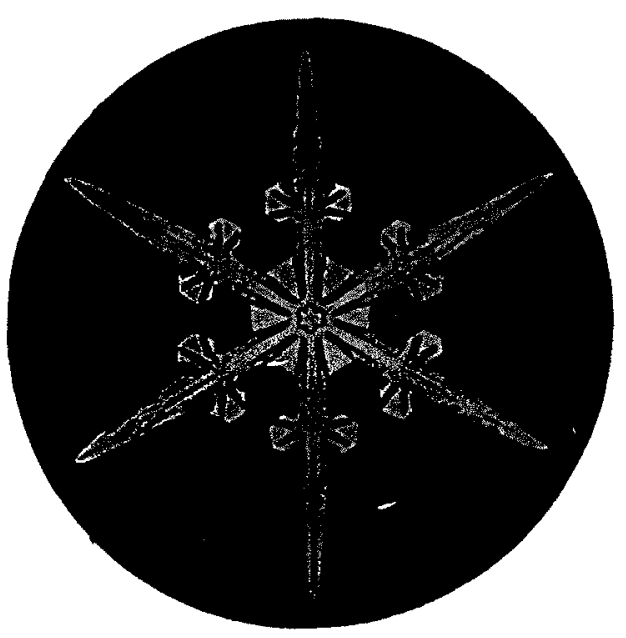

